\title{
Perspectivas
}

ISSN-L: 2215-4728

DOI: http://dx.doi.org/10.15359/rp.22.2

http://www.revistas.una.ac.cr/perspectivas

N. ${ }^{\circ} 22$. Enero-Junio, $2021 \cdot$ pp. 1-19

\section{Joaquín Prats: la didáctica de las ciencias sociales como ciencia}

\author{
Joaquín Prats: the Didactics of Social Sciences as \\ Science
}

\section{Arturo Meléndez Montero*}

Fecha de recepción: 15/02/2021 • Fecha de aceptación: 14/03/2021

Resumen: Definir el estatus epistemológico de la disciplina didáctica conlleva una serie de problemáticas que se intensifican cuando se hace referencia a una didáctica específica, aún más cuando esta trabaja con los conocimientos generados por las ciencias sociales, de cuyas disciplinas se ha puesto muchas veces en cuestión su categoría de ciencias. En el presente texto, se expone cómo el historiador y didacta Joaquín Prats aborda el problema epistemológico en la didáctica de las ciencias sociales, utilizando para ello la teoría de la ciencia elaborada por el filósofo Mario Bunge, desde la cual justifica la

\begin{abstract}
Defining the epistemological status of the didactic discipline entails a series of problems that intensify when specific didactics is referred to, even more when it works with the knowledge generated by the social sciences, of whose disciplines its category has been questioned many times of science. In this text, it is exposed how the historian and didact Joaquín Prats addresses the epistemological problem in the didactics of the social sciences, using for this the theory of science elaborated by the philosopher Mario Bunge, from which he justifies the category of said discipline as science. In
\end{abstract}

* Costarricense. Bachiller en la Enseñanza de los Estudios Sociales y la Educación Cívica, por la Universidad de Costa Rica (UCR), Sede de Occidente, San Ramón, Alajuela, Costa Rica. Actualmente, cursa la Licenciatura en Enseñanza de los Estudios Sociales y la Educación Cívica en la Universidad Nacional de Costa Rica (UNA), Sede Central Omar Dengo, Heredia, Costa Rica y el Bachillerato en Filosofía en la Universidad de Costa Rica (UCR), Sede Central Rodrigo Facio, San José, Costa Rica. Investigador independiente.

Correo electrónico: arturo.melendez.montero@est.una.ac.cr.

ORCID: http://orcid.org/0000-0002-3448-4774. 
categoría de dicha disciplina como ciencia. Así, argumenta la relevancia de la aplicación rigurosa del método científico y de las particularidades metodológicas que la disciplina requiere. Por último, se explica la forma en la que Prats lleva su posicionamiento cientificista a la enseñanza de la historia, como fórmula para generar criterio en el estudiantado, frente a los fenómenos históricos.

Palabras claves: estudios sociales; ciencias sociales; epistemología; método científico; enseñanza de la historia. this way, he argues the relevance of the rigorous application of the scientific method and of the methodological particularities that the discipline requires. Finally, it is exposed how Prats takes his scientific position to the teaching of history, as a formula to generate criteria in students about historical phenomena.

Keywords: social studies; social sciences; epistemology; scientific method; teaching of history.

\section{Introducción}

El presente trabajo se comprende como un ensayo teórico que aborda el problema epistemológico en la didáctica de las ciencias sociales, desde la obra del Dr. Joaquín Prats Cuevas, y con respecto a su aporte en el desarrollo de dicha disciplina como campo de estudio independiente y autónomo en las ciencias educativas. Además, reflexiona sobre la argumentación epistemológica realizada por Prats para justificar su propuesta, la cual entiende la didáctica de las ciencias sociales como una ciencia que puede alcanzar niveles de rigurosidad equivalentes a los de las denominadas ciencias naturales, ya que "las posibilidades son idénticas, aunque las características sean diferentes" (Prats, 2002, p. 86). Como eje central de este análisis, se plantea la utilización que el autor realiza de la teoría bungeana de la ciencia y lo importante del método científico en la investigación de las didácticas específicas. También se expone la aplicación, que ejecuta el autor, de los postulados cientificis$\operatorname{tas}^{\wedge} \mathrm{F}$ en la didáctica de las ciencias sociales, específicamente, en la enseñanza de la historia.

Para llevar a cabo una reflexión en torno a la idea de la didáctica de las ciencias sociales como ciencia, elaborada por Prats, se estudiaron las siguientes obras del autor: Historia y epistemología de las ciencias. Hacia una definición de la investigación didáctica de las ciencias sociales y ¿Es posible una historia objetiva sobre la realidad social? Reflexiones básicas e imprescindibles para investigadores noveles. Con 
respecto al abordaje propiamente de la investigación en la enseñanza de la historia, como parte de la ciencia de la didáctica de las ciencias sociales, se utilizaron los textos Iberoamérica en las aulas, ¿Por qué enseñar historia? y Combates por la historia en la educación.

El presente tratamiento de la obra de Prats muestra y resume la argumentación epistemológica que dicho autor realiza para el campo de la didáctica de las ciencias sociales, evidenciando las diferencias que marca su posicionamiento cientificista con respecto a la posición epistemológica de otros autores claves en el desarrollo de la didáctica de las ciencias sociales, como Alicia Camilloni y Joan Pagès. Asimismo, permite plantear críticas, desde dicho posicionamiento, a la concepción de la enseñanza de la historia presente en los currículos educativos oficiales.

\section{Joaquín Prats y su labor en la didáctica de las ciencias sociales ${ }^{1}$}

Joaquín Prats es un intelectual español, historiador y didacta de la historia, quien labora principalmente para la Universitat de Barcelona ${ }^{2}$. A su vez, funge como director de los programas de Doctorado Didáctica de las Ciencias, las Lenguas, las Artes y las Humanidades, y de Didáctica de las Ciencias Sociales y del Patrimonio, por lo que se trata de un investigador muy reconocido en dichos campos. Igualmente, ha sido director de múltiples tesis y estudios vinculados.

Su obra se centra en los temas de didáctica de las ciencias sociales, enseñanza de la historia y patrimonio cultural. Cuenta con más de 270 publicaciones, las cuales ha elaborado o en las que ha participado. Reúne 12 libros (producidos tanto en lo individual como en equipo), así como 43 artículos científicos. Para efectos de este trabajo, interesan sus principales textos y su labor enfocada en la didáctica de las ciencias sociales.

1 La información sobre el autor, expuesta en este apartado, es tomada de su currículum disponible en la página de la Universitat de Barcelona.

2 Como formación académica, el autor posee un doctorado en historia moderna. Se especializó en didáctica de la historia con énfasis en el análisis de los sistemas educativos y en la enseñanza de la historia de las universidades. También, tiene otros títulos honoris causa, otorgados por su labor académica. 
Prats es uno de los pioneros del campo de la didáctica de las ciencias sociales, la que, según menciona el autor, se encuentra "en una fase muy embrionaria" (Prats, 2002, p. 81). Su obra es de gran relevancia, debido a que otorga un lugar a dicha disciplina en la academia y las investigaciones científicas. Sumado a esto, su trabajo divulgativo e informativo ha servido también para llevar la investigación en didáctica de las ciencias sociales a Latinoamérica, no solo por medio de sus textos, sino por su papel como director del programa Formación del Profesorado de Educación Secundaria de Ecuador; en este país, ha sido el principal gestor del estudio de las didácticas específicas y, especialmente, de la didáctica de las ciencias sociales y la enseñanza de la historia.

\section{Epistemología y ciencia en la didáctica de las ciencias sociales}

Uno de los principales conflictos de la disciplina didáctica es el de su calidad epistemológica, principalmente no cuando se hace referencia a la didáctica en general, que de por sí ya es problemática, sino cuando se habla de una didáctica específica. Las dificultades surgen a la hora de pensar en esta como un quehacer científico y el panorama se complica cuando la didáctica específica que se encuentra en cuestión se basa en las denominadas ciencias sociales, cuya categoría de "ciencias" ha sido puesta en discusión, en diferentes ocasiones, en la historia de la epistemología científica.

Sobre esto se refiere Camilloni (2013), quien busca dar espacio a la didáctica de las ciencias sociales como ciencias, mediante un recorrido por las principales corrientes epistemológicas, en el que realiza un abordaje, primeramente, del positivismo y el neopositivismo. Estas corrientes, según la autora, buscan la correspondencia máxima de las teorías científicas con la realidad, centrándose en el qué y despreciando el para qué, visto este último como un obstáculo en la objetividad científica. Sobre esto, menciona: "el interés y la inclinación son expulsados de la ciencia. $\mathrm{O}$ son apartados, al menos cuando no responde a lo que se establece como racionalidad científica" (Camilloni, 2013, p. 28).

Con este principio, el positivismo busca alejar los juicios subjetivos del quehacer científico, lo que pondría a todos los estudios, acerca de los aspectos relacionados con "lo social", fuera del ámbito científico, 
ya que estos están mediados por el contexto de quien los realiza, pues la subjetividad del mismo investigador es parte del objeto en estudio; en la misma postura colocaría a los estudios en educación, entendiéndose esta también como una práctica social. Ante dicha problemática, Camilloni (2013) encuentra solución para conceptualizar las ciencias sociales y la didáctica como ciencias, en los postulados de la teoría crítica de Frankfurt, principalmente en autores como Habermas, Adorno y Horkheimer, para quienes el conocimiento científico está estrechamente relacionado con los fines de los que investigan "la realidad".

Esa posición tomada por Camilloni (2013) supone una relevancia de la teoría sobre la rigurosidad del método, aunque no llama a un relativismo científico. La siguiente cita de Adorno, que la autora expone, resume en buena medida su concepción epistemológica de la ciencia:

la anticipación del conjunto estructural constituye el primer paso en la comprensión de la realidad. La teoría tiene que descomponer la consistencia del objeto en un campo de tensión, de lo real y lo posible, puesto que este último no existe sin aquél. La anticipación de la unidad del todo constituye la premisa inalienable de todo análisis de los fenómenos sociales (Adorno en: Camilloni, 2013, p. 32).

La idea de la teoría como articuladora de la intencionalidad que anticipa el abordaje del objeto supone, por tanto, la tensión entre "lo real y lo posible", es decir, entre el qué y para qué de la investigación científica; existe, así, un sesgo de subjetividad inherente a todo estudio científico y, por ende, a la construcción del método. Por ello, la posición de Camilloni (2013) no desprecia la investigación empírico-analítica en las ciencias sociales, sino que la concibe como la materia prima para interpretar lo propiciado por la teoría.

Tal concepción epistemológica es compartida por Santisteban y Pagès (2018), en un texto que aborda los postulados de Camilloni, titulado La didáctica de las ciencias sociales y sus disciplinas. Reflexiones al hilo de la obra de Camilloni. En él, los autores mencionan que "Separar los hechos de los valores, por ejemplo, implica correr el riesgo, «de enseñar a los estudiantes cómo abordar el problema de los medios divorciándolos de la cuestión de los fines», tal como señala Giroux (1990, 
109)" (Santisteban y Pagès, 2018, p. 55). La idea del enlace entre la cuestión y los fines en la didáctica de las ciencias sociales está presente en la obra de Pagès, a partir de la propuesta de un currículum crítico, que propone supeditar los contenidos y técnicas al fin emancipador de la educación; dicho posicionamiento se basa en la teoría de la pedagogía crítica, principalmente desde las propuestas de estudiosos como Giroux, Apple y Popkewitz (Pagès,1994).

Sin embargo, ante estas discusiones presentadas por Camilloni y Pagès, Prats encuentra otra solución para la problemática de si se puede concebir la didáctica de las ciencias sociales como un campo científico. Su solución es, tal vez, de mayor complejidad metodológica, pues no se aleja del pensamiento cientificista que distingue la objetividad del saber producido por la ciencia de los otros tipos de conocimientos no científicos, sin caer en las discriminaciones y reducciones tanto del positivismo como del neopositivismo. Dicha solución es lograda por Prats, a partir de los postulados de la teoría de la ciencia del filósofo argentino Mario Bunge, principal referente epistemológico en la obra del didacta español de las ciencias sociales.

\section{La concepción bungeana de la ciencia como fundamento episté- mico en la obra de Prats}

Como se mencionó, el fundamento epistemológico desde el cual parte Joaquín Prats para desarrollar su propuesta de didáctica de las ciencias sociales como campo de lo científico es la filosofía de la ciencia manejada por Mario Bunge, lo que se expresa tanto explícita como implícitamente en la obra del didacta. Esto le da un carácter metodológico cientificista a su concepción de la didáctica de las ciencias sociales, al compartir la noción bungeana de la ciencia como "un estilo de pensamiento y acción: precisamente el más reciente, el más universal y el más provechoso de todos los estilos" (Bunge, 2004, p. 3).

Esta idea de la ciencia como un estilo de pensamiento y acción le permite a Prats no solo reconocer que existen otros modos de conocimientos y procesos de elaboración, sino también que la ciencia tiene un 
elemento de praxis, por lo que está mediada por fines preestablecidos. Así, se muestra, con base en Bunge, un carácter dialéctico en la construcción del conocimiento: "el conocimiento es el producto final de una relación dialéctica, permanente y necesaria entre el interior racional y cognoscitivo de los seres humanos y el mundo exterior espacial-temporal en el que se desenvuelven" (Bunge citado en Prats y Fernández, 2017, p. 98).

La ciencia sería, entonces, para Bunge y, por lo tanto, para Prats, un tipo de conocimiento que se construye y se relaciona con lo denominado "conocimiento común", o no científico. En este último, se podrían ubicar los fines prestablecidos, pero dicho postulado lleva a la pregunta: ¿qué hace a un conocimiento científico? La respuesta que se da a partir de la filosofía de Bunge es el método, lo que se expresa en la frase "El método científico es un rasgo característico de la ciencia, tanto pura como de la aplicada: donde no hay método científico no hay ciencia" (Bunge, 2004, p. 11).

El método científico es, de tal modo, el proceso por medio del cual se crea el conocimiento. Dicho método tendría, desde la concepción bungeana, una serie de pautas que le correspondería aplicar a cualquier disciplina de calidad de científica; este argumento es uno de los principales expuestos por Prats para justificar la idea de las ciencias sociales como propiamente ciencias. A causa del cumplimiento de las fases aludidas, el investigador menciona que "el término método es empleado, en un sentido general, para definir el conjunto de operaciones generales, comunes a cualquier ciencia: inducción y deducción, análisis y síntesis, planteamiento de hipótesis y su comprobación, etc.” (Prats, 2002, p. 86).

El autor considera que la definición bungeana del método permite, a su vez, considerar variaciones y especificidades que darían paso a un método o metodología particular, la cual cada disciplina elabora según su objeto y naturaleza de estudio. Por ello, parte de la siguiente idea basada en Pizarro $(1998)^{3}$, para identificar y justificar la diversidad, o bien pluralidades del método en las disciplinas sociales:

3 Y sus ideas expuestas en el texto Tratado de metodología de las ciencias sociales, en donde Pizarro (1998) analiza y expone la diversidad metodológica de las ciencias sociales (principalmente de la sociología), según el objeto y el desarrollo histórico de cada una de estas: 
Pero las particularidades específicas del objeto de estudio que abordan las distintas disciplinas sociales les obligan no a tener un método específico - por lo demás, es el mismo que el de la ciencia en general - , sino unas determinadas metodologías particulares de estudio según la parcela concreta de lo social que cada disciplina aborde (Prats, 2002, p. 86).

Esta generalidad y particularidad del método posibilita, tal como se mencionó, formular la concepción de Prats sobre las ciencias de lo social como genuinas, sin desprenderse de la rigurosidad metodológica que deja realizar la verificación ${ }^{4}$, o bien refutación de un enunciado teórico, entre las teorías científicas con respecto a la realidad objetiva y experimentable, es decir, sin restarle importancia al método. Así, se puede interpretar desde los enunciados de autores clásicos en el tema, como Adorno en Epistemología y ciencias sociales; esto sin limitar el carácter de científico a únicamente las ciencias físicas, como lo proponen el positivismo y neopositivismo.

\section{La didáctica de las ciencias sociales como campo científico de la educación}

A partir de lo expuesto, se puede tratar de forma específica el campo de la didáctica de las ciencias sociales como la ciencia autónoma

no pienso que exista ningún modelo general de toda investigación científica, ninguna lógica del descubrimiento que sólo con aplicar a un campo de lo real produzca automáticamente ciencia: la única epistemología posible es, para mí, aquella que se funda en la historia de las ciencias y que de ella aprende, en el mejor de los casos, lo que no es posible hacer. La reflexión sobre la historia de las ciencias nos enseña, en efecto, cómo se han ido construyendo y modificando los objetos, métodos y técnicas de las diferentes ciencias (Pizarro, 1998, p. 13).

4 El concepto de verificación en Bunge tiene algunas distinciones con respecto a los términos de falsación-corroboración popperianos, esto lo expresa en Las ciencias sociales en discusión. Una perspectiva filosófica:

a diferencia de Popper (1959), yo no equiparo «verificabilidad» con «refutabilidad» (véase Bunge, 1998). Desde luego, los contraejemplos, si se corroboran repetidas veces, debilitan y hasta falsan las hipótesis generales. Pero queremos algo más que la mera erradicación de las falsedades. También ambicionamos conocer la verdad, y esto exige una evidencia positiva. Por ejemplo, como «aún no descubierto» no es lo mismo que «inexistente» (...) En resumen, la confirmación es tan valedera como la refutación. (En contraste, la refutabilidad tiene más importancia que la conformabilidad.) Esto vale tanto para la historia como para la física (véase Bunge, 1967a, 1973a, 1985b y 1996a) (Bunge, 1999, p. 314). 
que plantea Prats. Lo anterior es capaz de resumirse en la siguiente frase de Prats: "el método científico será el criterio de demarcación fundamental del conocimiento científico; el resultado del estudio de todo objeto observable al que, susceptiblemente, podamos aplicar dicho método puede merecer el calificativo de científico" (Prats, 2002, p. 86). La idea expresa totalmente la concepción de ciencia y la posibilidad que encuentra Prats para elaborar un criterio sobre las ciencias de lo social; en este sentido, concibe la educación como un quehacer social, y, por ende, con la oportunidad de entenderse aquellas como ciencias a partir del método.

Al concebir de tal manera la alternativa de una ciencia de la educación, surge la problemática de si es posible otorgar esta categoría de "ciencia educativa" a una didáctica específica, como la de las ciencias sociales. Para ello, acorde con lo mencionado, se necesitaría la aplicación del método científico en dicho campo y la definición de una metodología específica que permite el abordaje de la particularidad de su objeto de estudio; esto último, según Prats (2002), en referencia a Estany (1990), dependería de los siguientes factores:

en primer lugar, del momento evolutivo en el que se encuentre el método general de la ciencia en cada etapa histórica; en segundo lugar, del grado de acumulación de conocimientos; en tercero, de la naturaleza del problema que el investigador esté estudiando; y, por último, del grado de interdisciplinariedad que la ciencia específica en cuestión pueda tener con otras afines (Prats, 2002, p. 86).

Esos factores definirían el grado de madurez metodológica de una disciplina científica. Lo anterior, aplicado a la didáctica de las ciencias sociales, habilita a Prats a ubicar al campo de estudio en cuestión en un estado "embrionario", con respecto a otras ciencias sociales y, aún más, a las ciencias naturales; sin embargo, para el autor dicha aseveración no determina la imposibilidad epistemológica de desarrollar un campo al nivel de otras ciencias, teniendo clara la naturaleza de esta como didáctica, la cual define:

como un campo de investigación educativa, situado en la encrucijada de diversas disciplinas entre las que están, por un lado, las que se 
ocupan tradicionalmente del aprendizaje y la enseñanza y, por otro, las que constituyen la base de los conocimientos que se pretenden enseñar (Prats y Fernández, 2017, p. 108).

Manifestado de otro modo, la didáctica de las ciencias sociales se encuentra en un espacio epistemológico medio, en el cual se ve influenciada por las disciplinas sociales que constituyen sus contenidos por enseñar, así como por la didáctica general, que aporta métodos y herramientas para transmitir dichos contenidos. Partiendo de esta noción de la didáctica como un campo de las ciencias educativas, Prats considera que, para alcanzar un desarrollo científico en la didáctica de las ciencias sociales, es necesario tener en cuenta lo anotado a continuación:

a. Considerar el requisito de que los procesos de indagación, observación, experimentación, etc. se basen en modelos y diseños que contengan rigor metodológico, partiendo de la pluralidad metodológica de las ciencias sociales.

b. Definir la finalidad, la intencionalidad y las líneas de investigación, con la aspiración de poder.

c. Elaborar teorías explicativas que sirvan para conocer los diversos agentes que intervienen en el proceso didáctico y las relaciones e interacciones entre estos agentes.

d. Definir los instrumentos que hacen referencia a las metodologías y representaciones pedagógicas.

e. El diseño, la experimentación y la evaluación de estrategias didácticas y la creación de materiales y elementos que sirvan para intervenir de manera controlada en el proceso de enseñanza y aprendizaje (Prats, 2002, p. 88).

La exigencia de estos criterios es, siguiendo a Prats, lo que fundamenta una propuesta científica de la didáctica de las ciencias sociales, reservada únicamente para lo correspondido con la praxis investigativa, pues esta rigurosidad del método que se expresa en el anterior punto "a", de acuerdo con él, no se presenta en la actividad didáctica, propiamente de la enseñanza, como el acto de la transmisión de conocimiento. Dicho 
punto llevaría al autor a rechazar la idea de la "investigación acción" en el aula como indagación de carácter científico, debido a que esta supone relativizar el método que omite el estricto cumplimiento de los "requisitos mínimos", el cual debe poseer toda investigación científica. ${ }^{5}$

El punto "b" aborda el dilema ideológico de la investigación científica, en el que se interrelaciona el qué se investiga con el para qué y, según el autor, deben estar en total correspondencia. El punto "c" hace referencia a la necesidad de la didáctica de las ciencias sociales de elaborar sus propias teorías como didáctica específica; dicho de otro modo, a las particularidades que la metodología del campo exige. El punto " $\mathrm{d}$ se refiere a la aplicación de los instrumentos; aquí Prats se destaca por la relevancia dada a la rigurosidad, lo que distingue su posición cientificista basada en Bunge de autores como Camilloni (cuyo posicionamiento se fundamenta en Adorno). El punto "e" remite al producto científico, a la puesta en práctica de los conocimientos extraídos de la investigación en el campo de la didáctica de las ciencias sociales.

Por último, Prats comprende que el desarrollo de las didácticas de las ciencias sociales como campo científico autónomo es una tarea en realización por parte de diversos investigadores. Por tanto, es necesario que, actualmente, se genere un espacio inclusivo, el cual busque integrar eclécticamente los diferentes planteamientos y posiciones, sin obviar las diferencias e incoherencias de fondo que en el mismo campo investigativo se puedan presentar (Prats, 2002).

5 Los requisitos mínimos de los que hace referencia Prats (2002) son los que Bunge (2004) denomina reglas del método científico: R1: Formular el problema con precisión; R2: Proponer conjeturas precisas y fundamentadas; R3: Someter las hipótesis a una contrastación dura; R4: No declarar las hipótesis como verdaderas, sino confirmarlas como parcialmente verdaderas; R5: No limitarse a las generalizaciones que se adecuen a los datos, sino buscar explicaciones a base en leyes. Esto parte desde los principios del método científico, que son para Bunge (2004): Formular preguntas problema precisas, configurar hipótesis sobre dichos problemas, ejecutar técnicas de contrastación, evidenciar, estimulación de las hipótesis y generación de un nuevo problema. 


\section{La enseñanza de la historia y la didáctica de las ciencias sociales según Prats}

En este eje se analiza la posición que ha enmarcado el pensamiento de Joaquín Prats, en torno a la enseñanza de la historia y las ciencias sociales. Como primer punto, se abordará el debate que plantea el investigador sobre la cientificidad de la historia y la evolución que ha tenido su enseñanza. Para otros didactas de las ciencias sociales, como Joan Pagès, la evolución historiográfica ${ }^{\wedge} \mathrm{F}$ que supone el abandono de una historia total, la fragmentación histórica y, principalmente, la diversidad tanto epistemológica como metodológica son de gran importancia en la enseñanza de la historia, ya que logran "proyectar una historia más humana que hable de todas las personas, pero será vista en algunos casos como un retroceso en la tarea de la comprensión global de las experiencias y los procesos humanos" (Massip, Castellvi y Pagès, 2020, p. 172). Según Prats (2016), la evolución historiográfica que se ha enmarcado en la corriente posmoderna es, precisamente, un retroceso en el desarrollo científico de la historia y la enseñanza ${ }^{6}$.

\section{El debate de Prats respecto a la enseñanza de la historia}

La enseñanza de la historia se encuentra en un continuo retroceso en el sentido de que en la mayoría de países occidentales se han eliminado contenidos y se ha ido transformando la historia como asignatura; se convirtió en una materia en la cual se aborda una serie de aspectos que provienen de otras disciplinas. De esta forma, no se analizan ni se abordan en sí procesos históricos, por el contrario, se crea una concepción de

6 La posición de Prats con respecto a la corriente posmoderna en la historia es contraria a la presentada por Pagès; esto es expuesto explícitamente en la siguiente cita:

Ha influido también en el retroceso y repliegue de la historia en su consideración como ciencia la ofensiva posmoderna que niega la posibilidad del conocimiento social obtenido a través del método científico. La tesis posmoderna ha negado esta posibilidad. Lo que llamamos ciencia social, según Lyotard (1989), constituye simplemente en un juego del lenguaje o, mejor, una pluralidad de juegos del lenguaje creado por los científicos sin otro criterio de legitimidad que el consenso de los que participan. Ello deslegitima profundamente el carácter científico de la tarea de los historiadores y de las ciencias de la educación (Prats, 2016, p. 152). 
la historia en la que solamente se enseñan conceptos o fechas y no tiene ningún grado de utilidad.

Según menciona Prats (2016), en el momento en que la historia pasó a ser un elemento trascendental para la consolidación de los Estados liberales y el surgimiento de los nacionalismos, esta historiografía trascendió a todos los niveles educativos. Su practicidad como herramienta de ideologización política la coloca en el debate actual. La evolución llevó a la historia a configurarse como una disciplina académica-científica, lo que se contrapone a las concepciones tradicionales de su enseñanza.

De acuerdo con Prats, Valls y Miralles (2015), los discursos en la enseñanza de la historia que contribuyeron a la formación de los nacionalismos transcienden al contexto iberoamericano. En el análisis realizado por los autores en Argentina, México, Ecuador y Brasil, se concluye que los libros de texto responden a una historia oficial; por ejemplo, del "descubrimiento de América" se pasa directamente a la independencia, es decir, no hay, en sí, procesos históricos.

Asimismo, un elemento fundamental que debe estar en la enseñanza de la historia es esa continuidad, mediante la que el pasado permite comprender el presente. Sin embargo, los resultados del estudio realizado llevado a cabo en Iberoamérica evidencian que dicha continuidad no está en los manuales curriculares, ni dichos elementos en los libros de texto. Aunado a esto, un señalamiento que realiza el autor es la ausencia de una dimensión iberoamericana en enseñanza de la historia; por tanto, se arrastra una postura eurocéntrica que limita la existencia de un concepto regional de la historia. Es por ello que se recalca la importancia de reivindicar tal disciplina desde la cientificidad, es decir, considerar aquella historia que es crítica y meticulosa, la cual recurre al método científico; para Prats, surge como una necesidad el visualizarla desde su configuración científica, para "educar la conciencia colectiva de los ciudadanos" (Prats, 2015, p. 3).

Por otra parte, Prats (2015) también analiza cómo en la actualidad la educación juega un papel importante, con miras a fortalecer el modelo de mercado actual. Así, bajo la mirada de los organismos internacionales como el Fondo Monetario Internacional, el Banco Mundial o la OCDE, la historia ha sido invisibilizada; dejó de ser una asignatura elemental y 
como ejemplo de ello se refiere a la reciente exclusión de las ciencias sociales de las pruebas instrumentales y los informes PISA.

En la misma línea descrita, el didacta considera que se debe abordar la historia como un conocimiento social y, por ende, como materia educativa con un rol de transformadora de la sociedad. Lo anterior, porque la disciplina permite comprender la realidad y estimular el desarrollo del pensamiento crítico, por medio de la que los estudiantes puedan tener capacidad de discernir sobre temas políticos y socioeconómicos. En este sentido, Prats señala que la enseñanza de la historia debe instar al análisis de lo compleja que resulta la realidad.

Prats también recalca la importancia del pensar históricamente. Con ello, sugiere que, al momento de enseñar historia, el estudiante debe aprender a criticar y validar las evidencias de un fenómeno. Igualmente, hace referencia a la historia no como un conjunto de conocimientos acabados, sino como aquella que debe adaptarse a las dinámicas del estudiantado. El autor no plantea cambiar la historia, sino presentarla de una manera que, en metodología, sea interesante y trascendental para el alumno; es decir, la disciplina consiste en convertir la narración en un problema, buscando que los y las estudiantes sepan construir productos históricos (Prats, 2001).

El método de enseñanza de la historia del investigador en cuestión marca, de nuevo una, diferencia con el posicionamiento de Pagès (2018). Para este último, debe existir una distinción, en cuanto a fines y métodos, entre el conocimiento historiográfico de la academia y la historia escolar: la segunda se debe ocupar del fomento, en los alumnos, acorde con Mattozzi (2015), de la interpretación crítica de los fenómenos históricos, sin la necesidad de llevar al aula la rigurosidad del método científico, como lo plantea Prats.

Para Prats (2015), la enseñanza de la historia en el siglo XXI debe surgir desde las gentes y las sociedades, por tanto, debe direccionarse hacia tres objetivos globales: perspectiva, conocimiento y competencia. Con respecto a la perspectiva, el autor plantea que dicha enseñanza de la disciplina estudia la causalidad de los fenómenos históricos y, a su vez, explica los problemas sociales. En cuanto al conocimiento, el investigador señala que la didáctica de la historia debe procurar el saber de una serie de conceptos, pero desde una perspectiva de cambio y continuidad, 
es decir, que los conocimientos no se estudien de manera aislada. Finalmente, respecto a las competencias que debe generar la historia en el aula, el escritor sugiere que la didáctica de la historia debe simular el trabajo del historiador en el espacio educativo, con el fin de que los estudiantes la construyan incluyendo competencias de sensibilidad.

Lo previo debe comprenderse desde la influencia del pensamiento piagetiano en Prats; con esta noción, argumenta que la enseñanza de la historia es preciso conformarla bajo un sistema de pensamiento, mediante el cual, poco a poco, el estudiante pueda interiorizar la complejidad de los sistemas e ir formando su propio criterio de la realidad social. El aporte fundamental de Prats alrededor de la enseñanza de la historia es que, al considerarla ciencia social, esta debe procurar el desarrollo del pensamiento crítico y, de esta manera, alejarse de la concepción tradicional de la historia escolar, que responde ciegamente a los nacionalismos (Prats, 2015).

\section{Hacia una ciencia integral de la didáctica de las ciencias sociales}

Como se ha mencionado, Prats plantea la enseñanza de la historia como ciencia social, no obstante, el autor analiza esa postura de cientificidad en torno a la didáctica de las ciencias sociales; sugiere que debería consolidarse como un campo propio de investigación nutrido de otras ciencias sociales como geografía, historia, entre otras. Anudado a lo anterior, entiende que la didáctica de las ciencias sociales debe enmarcarse en el campo de la investigación educativa, en el sentido del porqué y para qué enseñar. También identifica la necesidad de replantearse metodológicamente el funcionamiento de las didácticas de las ciencias sociales, procurando unir su intencionalidad y sus líneas de indagación, idea que se desprende de los criterios requeridos para la cientificidad en la didáctica de las ciencias sociales, ya expuestos.

De este modo, la didáctica de las ciencias sociales no puede limitarse en sí a una disciplina, por lo que el sentido de una línea de investigación de las ciencias sociales consiste en que esta se plantee un cambio significativo en la formación del profesorado, en las vías de aprendizaje de los y las estudiantes e incluso en la propia conceptualización de las ciencias sociales (Prats, 2001). Así, la problemática sobre dónde 
posicionar la didáctica de las ciencias sociales ha repercutido directamente en la formación de los alumnos.

Por su parte, Gómez y Rodríguez (2014) comentan que la mayoría de las docentes en España se especializa en una disciplina en específico o se concentra en el campo de la educación. Este mismo aspecto lo rescata Prats (2015) para el caso de América Latina: aduce que la didáctica de las ciencias sociales como planteamiento científico-académico no se logra en la mayor parte de los espacios educativos, debido a que no hay claridad sobre ellas.

Ahora bien, Gómez et al. (2014) hacen referencia a la obra de Prats y concuerdan con que la intencionalidad de las ciencias sociales dentro de las aulas es de formar competencias para comprender la realidad social. Con base en esta premisa, cada contenido debe visualizarse partiendo de las diferentes disciplinas que nutren las ciencias sociales. Por tanto, es fundamental que desde el espacio educativo se visualicen esas líneas de investigación en la didáctica de las ciencias sociales, con miras a construir conocimientos que van más allá de los contenidos teóricos y conceptuales.

\section{Conclusiones}

En el presente trabajo, se reflexionó sobre diversas obras del teórico de la didáctica de las ciencias sociales, Joaquín Prats. En un primer apartado, se abordan sus aportes epistemológicos, los cuales buscan consolidar una argumentación científica de la didáctica de las ciencias sociales. En el segundo segmento, se expone cómo Prats lleva a la aplicabilidad dicha argumentación, para generar una propuesta en la enseñanza de la historia y la didáctica de las ciencias sociales. De ambos apartados, se derivan las siguientes conclusiones:

1. El planteamiento de Prats propone una idea de las didácticas de las ciencias sociales como un campo científico y autónomo en las ciencias educativas, con posibilidad de lograr niveles de rigurosidad científica, equivalentes a los de las ciencias naturales. Dicha propuesta, parte de la filosofía bungeana de la ciencia, en la cual 
se le da una importancia categorial al método como la fórmula que hace la ciencia.

2. En relación con el primer punto, es importante estudiar la distinción entre las nociones de método científico general y método particular, o metodología; este último hace referencia a las particularidades que se presentan, según el objeto de estudio, en las diversas disciplinas sociales. Este aspecto supone, por tanto, la necesidad de desarrollar un método de investigación propio en las didácticas de las ciencias sociales.

3. La didáctica de las ciencias sociales es entendida por Prats como un "campo embrionario" de las ciencias en general, pero esto no limita su posibilidad de desarrollo, para el cual es necesario perseguir los siguientes criterios: establecer un método propio, una comunidad científica, teorías propias del campo que estén en relación con el contexto educativo; tener la intencionalidad de que el estudiante desarrolle las competencias para comprender su realidad críticamente.

4. Para Prats, a diferencia de otros teóricos de la didáctica de las ciencias sociales, la historia ha venido en un proceso de decadencia, por la pérdida de la rigurosidad científica producto de los discursos posmodernos, lo que a su vez ha ubicado la enseñanza de la historia como una disciplina de segunda categoría en el currículum educativo, al ya no responder a los intereses hegemónicos de los Estados nacionales. Estas afirmaciones exigen la necesidad de simular el método científico en el aula, mediante estrategias didácticas, para propiciar el desarrollo de una historia científica y cuestionar la historia tradicional presente en los currículums educativos.

De forma general, se observa que los aportes de la obra Prats han sido y siguen siendo importantes para la comprensión de la didáctica de las ciencias sociales y de la enseñanza de la historia. Sus textos exponen una argumentación que permite concebir la didáctica de las ciencias sociales como un campo científico definido y, aunque se entienda este 
como un "embrión" en crecimiento en relación con otras ciencias, posee relevancia en la generación de conocimiento global.

La posición cientificista de Prats representa una vía epistemológica que contrasta con los postulados de otros teóricos de la didáctica de las ciencias sociales, tales como Alicia Camilloni y Joan Pagès. Ellos toman otros caminos epistemológicos que no tienen como base un principio de rigurosidad metodológica, sino la labor ideológica e interpretativa de la teoría. A diferencia de estos, el razonamiento planteado por Prats permite reivindicar la importancia de dicha rigurosidad del método, como el medio para generar en los estudiantes pensamiento crítico y otras competencias, los cuales les posibiliten comprender y hacer frente a los fenómenos sociales que acontecen.

\section{Bibliografía}

Adorno, T. (2001). Epistemología y ciencias sociales. Madrid: Ediciones Cátedra.

Bunge, M. (1999). Las ciencias sociales en discusión. Una perspectiva filosófica. Argentina: Editorial sudamericana.

Bunge, M. (2004). La investigación científica: su estrategia y su filosofía. Buenos Aires: Siglo XXI.

Camilloni, A. (2013). Epistemología de la didáctica de las ciencias sociales. En B. Aiserberg y S. Alderoqui (comps.), Didáctica de las ciencias sociales: aportes y reflexiones. Buenos Aires: Paidós.

Gómez, C. y Rodríguez R. (2014). Aprender a enseñar ciencias sociales con métodos de indagación. Los estudios de caso en la formación del profesorado. Revista de docencia universitaria, 12(22), 307-325.

Massip, M., Castellví, J. y Pagès, J. (2020). La historia de las personas: reflexiones desde la historiografía y de la didáctica de las ciencias sociales durante los últimos 25 años. Planta Rei. Revista digital de historia y didáctica de la historia, 14(2), 167-196. https://doi.org/10.6018/pantarei.445831.

Mattozzi, I. (2015). Una epistemología y una metodología para la didáctica, Diálogos, 19(1), 57-72. DOI: https://doi.org/10.4025/dialogos.v19i1.1033.

Pagès, J. (1994). La didáctica de las Ciencias Sociales, el currículum de la historia y la formación del profesorado. Signos. Teoría y práctica de la educación, (13), $38-51$. 
Pagès, J. (2018). Las relaciones entre la historia y la historia escolar. Trayectorias Universitarias, 4(7), 31-37.

Pizarro, N. (1998). Tratado de metodología de las ciencias sociales. España: Siglo veintiuno de España editores, S. A.

Prats, J., Valls, R. y Miralles, P. (2015). Iberoamérica en las aulas. España: Editorial Milenio. Lleida.

Prats, J. y Santacana, J. (2011). ¿Por qué y para qué la historia? En F. Rodríguez y N. García (orgs.), Enseñanza y aprendizaje de la historia en la educación básica (pp. 18-64). México: Secretaría de Educación Pública.

Prats, J. (2001). Enseñar Historia: Notas para una didáctica renovadora. España: Consejería de Educación, Ciencia y Tecnología. Mérida.

Prats, J. (2002). Historia y epistemología de las ciencias. Hacia una definición de la investigación didáctica de las ciencias sociales. Enseñanza de las ciencias sociales, (1), 81-89.

Prats, J. (2016) Combate por la historia en la Educación. Revista Enseñanza de las Ciencias Sociales, (15), 145-153. DOI: https://doi.org/10.1344/ECCSS2016.15.13.

Prats, J. y Fernández, R. (2017). ¿Es posible una explicación objetiva sobre la realidad social? Reflexiones básicas e imprescindibles para investigadores noveles. Retos y dificultades para la enseñanza de la historia. Didacticae, (1), 97-110. https://doi.org/10.1344/did.2017.1.97-110.

Santisteban, A. y Pagès, J. (2018). La didáctica de las ciencias sociales y sus disciplinas. Reflexiones al hilo de la obra de Camilloni. Revista de Educación,(12), 45-65.

Universitat de Barcelona. (2020). Currículum vitae de Joaquín Prats Cuevas. 